\title{
Force Balance Conditions of Complex Parallel Mechanisms with Mass Equivalent Modeling
}

\author{
V. van der Wijk \\ Centre for Robotics Research, Dep. of Informatics, Fac. of Natural and \\ Mathematical Sciences, King's College London, Strand, London (UK) \\ e-mail: Volkert.vanderWijk@kcl.ac.uk
}

\begin{abstract}
A shaking force balanced mechanism is a mechanism that does not exert dynamic reaction forces to its base and to its surrounding for any motion. For mobile mechanisms such as exoskeletons, humanoid robots, drones, and anthropomorphic hands force balance is an important property for, among others, their dynamic behavior, stability, safety, control, and low energy consumption.

For the design of force balanced mechanisms with multiple closed loops it can be a significant challenge to obtain the balance conditions, especially when the mechanism consists of closed loops that depend on other closed loops. In this paper it is shown how with mass equivalent modeling the force balance conditions can be derived of a complex multi-degree-of-freedom parallel mechanism with multiple closed loops of which one or more depend on other closed loops. It is shown how such a mechanism can be divided in mass equivalent linkages such as mass equivalent dyads and mass equivalent triads for which each can be analyzed individually with principal vectors and linear momentum equations.
\end{abstract}

Key words: Force balance, mass equivalent modeling, parallel mechanism, center of mass

\section{Introduction}

A shaking force balanced mechanism is a mechanism that does not exert dynamic reaction forces to its base and to its surrounding for any motion. The sum of the linear momenta of all moving elements of a force balanced mechanism is constant for all motion which most of the times implies that the center of mass (CoM) of the mechanism is in a stationary point in the base. Also the motion of a force balanced mechanism is not affected by any translational motion of the base, i.e. the base and the mechanism are dynamically decoupled for translational motion of the base.

For mobile mechanisms such as exoskeletons, humanoid robots, drones, and anthropomorphic hands force balance is an important property for their dynamic behavior [6], stability and control [2], safety, and ergonomics [5]. Since force balanced mechanisms are also statically balanced - gravity does not affect their motion, they lead to energy friendly actuation [3] and also to an increase of safety of large moving 
structures such as bridges [6]. For fast moving robotic manipulators force balance reduces the base vibrations such that cycle times can be shorter [10].

For the design of force balanced mechanisms with multiple closed loops it can be a significant challenge to obtain the general force balance conditions, especially when the mechanism consists of closed loops that depend on other closed loops. Common methods to derive the balance conditions require the explicit formulation of the closed-loop relations which then need to be included in the other equations where the linear dependent relations among the links need to be eliminated [1]. If at all possible, this leads to considerable efforts.

With mass equivalent modeling the loop closure relations can be considered implicitly. This has already shown to have potential to derive the force balance conditions of simple parallel mechanisms by balancing each arm individually $[4,11,12]$ and also for complex parallel mechanisms by the design of inherently balanced closed-chain linkage architectures $[7,6]$ and by the design of mass equivalent dyad and triad linkages $[8,9]$. The essence of this approach for complex linkages is that one or multiple links together are modeled with real and virtual equivalent masses, which subsequently are projected on the remaining open-chain linkage and included for analysis.

The goal of this paper is to show how with mass equivalent modeling the force balance conditions can be derived of a complex multi-degree-of-freedom (multiDoF) parallel mechanism with multiple closed loops of which one or more depend on other closed loops. It is shown how such a mechanism can be divided in mass equivalent linkages such as mass equivalent dyads and mass equivalent triads for which each can be analyzed individually. First a two-DoF force balanced planar parallel mechanism with three closed loops is presented. This linkage is divided in one mass equivalent dyad and two mass equivalent triads. Then the balance conditions of the mass equivalent dyad are explained and subsequently the balance conditions and design parameters of the mass equivalent triads are obtained.

\section{Two-DoF force balanced planar parallel mechanism with three closed loops}

Figure 1a presents a planar parallel linkage which has two-DoF motion and three closed loops of which one depends on the other two. This new mechanism consists of the two four-bar linkages $A_{0} A_{1} A_{2} A_{3}$ and $A_{4} A_{5} A_{6} A_{7}$, with common base link $A_{0} A_{3} A_{7} A_{4}$, and a dyad $B_{1} C_{1} B_{2}$ of which $B_{1}$ and $B_{2}$ are revolute pairs with the coupler links of each four-bar linkage. Where each four-bar linkage has a single independent closed loop, the dyad gives a third dependent closed loop following a path through each four-bar linkage. From the perspective of a driven parallel manipulator, both four-bar linkages can be moved individually with two actuators at the base whereby the motion of the dyad with joint $C_{1}$ as the end-effector is determined. Such a mechanism could be useful, for instance, as a manipulator on a service and inspec- 


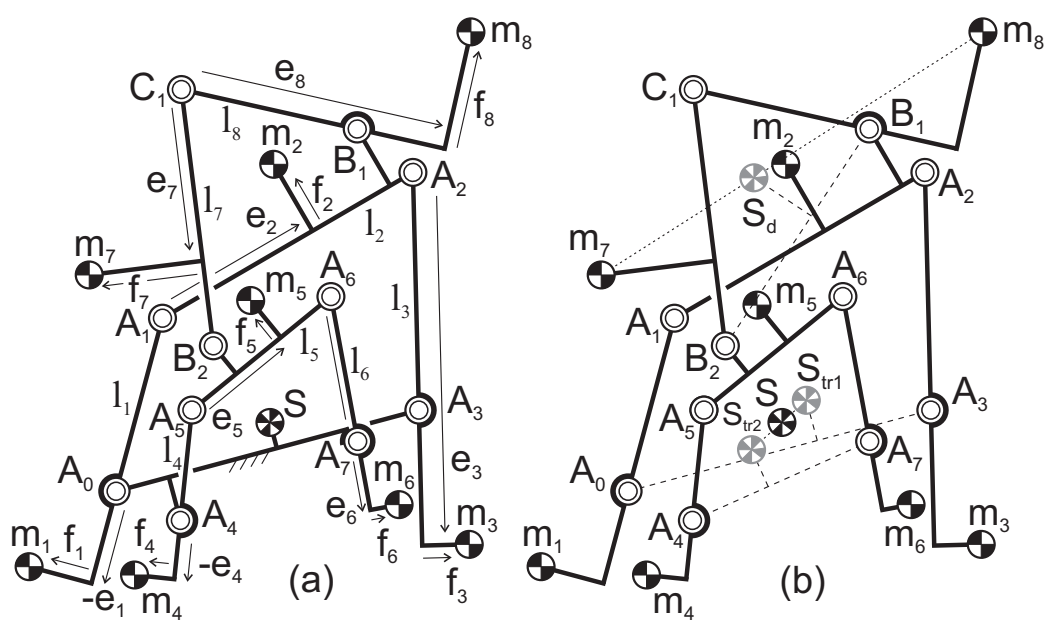

Fig. 1 a) Force balanced parallel mechanism composed of two 4R four-bar linkages with common base and a dyad pivoted with each coupler link (drawn to scale); b) The dyad and the two triads representing the $4 \mathrm{R}$ four-bar linkages are shown with their mass equivalent elements from which the force balance conditions are derived.

tion drone to move around rapidly without dynamically affecting (destabilizing) the hovering and manoeuvring drone itself.

Each link $i$ of the mechanism has a mass $m_{i}$ with its CoM defined by parameters $e_{i}$ and $f_{i}$ relative to the line through the joints of the link as illustrated in Fig. 1a. This means that each of the eight links can have a general design, i.e. mass symmetry is not required.

For specific relations among the link masses and the link CoMs the common CoM of all links together is in a stationary point $S$ in the base link for all motion of the mechanism. These relations are named the (shaking) force balance conditions which in fact are design criteria for the links. The mechanism in Fig. 1a is shown with one of the many force balance solutions and is drawn to scale for a realistic impression.

With common methods such as the linear independent vector method [1] it is specifically challenging to handle the closed loop by the dyad because of its dependency on the closed loops of each four-bar linkage. However with mass equivalent modeling this can be considered in a systematic and insightful manner.

To derive the general force balance conditions the mechanism can be divided in three parts which each then is analyzed by means of a mass equivalent model. These three parts are the dyad $B_{1} C_{1} B_{2}$ with links masses $m_{7}$ and $m_{8}$, and each of the two four-bar linkages. In the next section the dyad is investigated for mass equivalence and the four-bar linkages are investigated for mass equivalence in the subsequent section. 


\section{Mass equivalent dyad}

For force balance of the mechanism in Fig. 1a the dyad $B_{1} C_{1} B_{2}$ needs to have constant mass properties with respect to joints $B_{1}$ and $B_{2}$ for all motion such that these mass properties can be included in the force balance of the two four-bar linkages. This is since the motion of the dyad is nonlinearly related with the motion of the other links by which the design of the other links cannot contribute fully to the balance of the dyad links in another way. It can also be said that the dyad needs to be force balanced with respect to the 'imaginary dyad base link' $B_{1} B_{2}$. This imaginary link is shown in Fig. $1 \mathrm{~b}$ where $S_{d}$ is the common CoM of $m_{7}$ and $m_{8}$. Due to the motion of the mechanism the size of this imaginary link varies. As long as the triangle $B_{1} B_{2} S_{d}$ remains similar of shape for all motion while being scaled and rotated, the mass properties of the dyad relative to $B_{1}$ and $B_{2}$ are constant. The triangle $B_{1} B_{2} S_{d}$ with a mass $m_{7}+m_{8}$ in $S_{d}$ then is regarded a mass equivalent model of the dyad [8]. Also, from another viewpoint, for force balance the dyad needs to be mass equivalent to the model of the triangular element $B_{1} B_{2} S_{d}$ with mass $m_{7}+m_{8}$ in $S_{d}$.

The conditions for which the triangular element $B_{1} B_{2} S_{d}$ and the dyad are mass equivalent have been derived as [8]

$$
m_{7} e_{7}=m_{d}^{a} l_{7}, \quad m_{7} f_{7}=m_{d}^{c} l_{7}, \quad m_{8} e_{8}=m_{d}^{b} l_{8}, \quad m_{8} f_{8}=m_{d}^{c} l_{8}
$$

Here $m_{d}^{a}=\left(m_{7}+m_{8}\right)\left(1-\lambda_{d 1}\right)$ and $m_{d}^{b}=\left(m_{7}+m_{8}\right) \lambda_{d 1}$ are real equivalent masses with $m_{d}^{a}+m_{d}^{b}=m_{7}+m_{8}$ and $m_{d}^{c}=\left(m_{7}+m_{8}\right) \lambda_{d 2}$ is a virtual equivalent mass. $\lambda_{d 1}$ and $\lambda_{d 2}$ are the similarity parameters, i.e. the properties that define the shape of the triangle $B_{1} B_{2} S_{d}$ by describing the location of $S_{d}$ relative to line $B_{1} B_{2}$.

These conditions are the first four force balance conditions of the mechanism. For instance when $l_{7}, l_{8}, m_{7}, m_{8}, e_{7}$, and $f_{7}$ are given then $e_{8}$ and $f_{8}$ can be derived for force balance of the linkage in Fig. 1. Generally this means that the CoM of one of the dyad links is located beyond the joint with the coupler link as illustrated for $m_{8}$ that is located beyond joint $B_{1}$. In practice this implies the need of a countermass on link $B_{1} C_{1}$.

\section{Mass equivalent triads}

For force balance the two four-bar linkages need to have constant mass properties with respect to the base link $A_{0} A_{3} A_{7} A_{4}$, i.e. they need to be force balanced with respect to the base, for all motion. Since each four-bar linkage consists of three moving links, they can be regarded as triads $A_{0} A_{1} A_{2} A_{3}$ and $A_{4} A_{5} A_{6} A_{7}$ which, similar to the dyad, need to have constant mass properties with respect to their joints $A_{0}$, $A_{3}, A_{4}$, and $A_{7}$. This means that also the triads need to be mass equivalent to singleelement models [9]. This is illustrated in Fig. $1 \mathrm{~b}$ where triangle $A_{0} A_{3} S_{t r 1}$ represents the equivalent mass model of triad $A_{0} A_{1} A_{2} A_{3}$ and triangle $A_{4} A_{7} S_{t r 2}$ represents the equivalent mass model of triad $A_{4} A_{5} A_{6} A_{7}$. 


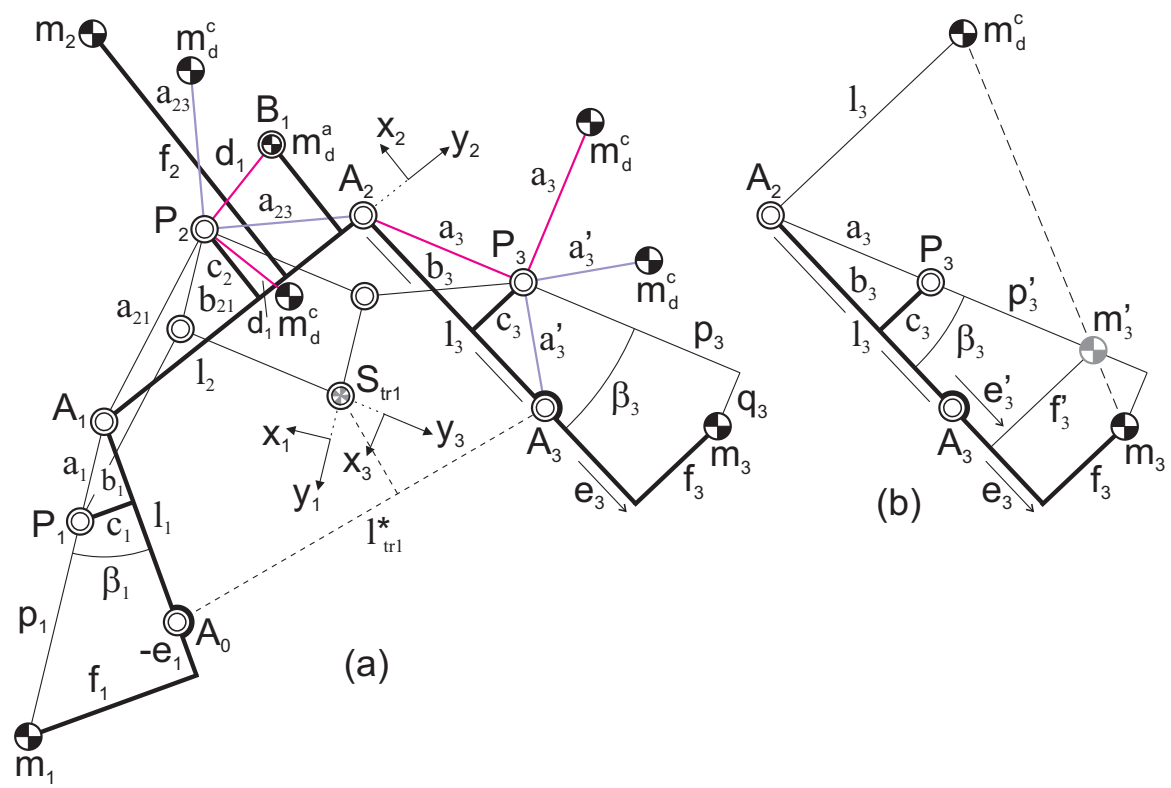

Fig. 2 a) Triad 1 as a 3-DoF principal vector linkage with mass projection of the equivalent dyad with $m_{d}^{a}$ in $B_{1}$ and $m_{d}^{c}$ about $P_{2}$ and $P_{3}$; b) For analysis of DoF 3 the masses in link $A_{2} A_{3}$ can be combined as $m_{3}^{\prime}$.

Since the dyad is located on top of the two triads, the mass of the dyad needs to be included in the triads as well. This means that the mass model $A_{0} A_{3} S_{t r 1}$ includes the mass of triad $A_{0} A_{1} A_{2} A_{3}$ and part of the dyad mass with their common CoM in $S_{t r 1}$ and that the mass model $A_{4} A_{7} S_{t r 2}$ includes the mass of triad $A_{4} A_{5} A_{6} A_{7}$ and the other part of the dyad mass with their common CoM in $S_{t r 2}$. Both mass models lay in the base link with the common CoM of $S_{t r 1}$ and $S_{t r 2}$ located in $S$, which is the common CoM of the complete mechanism.

The force balance conditions of the triads can be derived with the methodology presented in $[9,8,6]$ by analyzing each DoF of the triad independently with principal vectors. First these principal vectors are investigated, then the balance conditions are obtained with linear momentum equations for each relative DoF individually followed by the calculations of the mass parameters of the triad from the mass equivalent model.

Figure 2a shows the triad $A_{0} A_{1} A_{2} A_{3}$ as a 3-DoF principal vector linkage with a principal point $P_{1}, P_{2}$, and $P_{3}$ in each of the three principal elements - the triad links. These principal points define together with the principal joints $A_{1}$ and $A_{2}$ the three illustrated parallelograms which trace the common CoM in $S_{t r 1}$ for all motion. The lengths of the sides of the parallelograms $a_{1}, a_{21}, a_{23}$, and $a_{3}$ are the principal dimensions which are constants. This means that the parallelograms can be seen as rigid-body linkages with revolute pairs moving along with the triad. The location 
of the principal point in each principal element is defined with parameters $b_{i}$ and $c_{i}$ relative to the lines through the joints of the links.

In addition to the masses $m_{1}, m_{2}$, and $m_{3}$ of the triad, in Fig. $2 \mathrm{a}$ also the equivalent masses $m_{d}^{a}$ and $m_{d}^{c}$ of the dyad are projected. The real equivalent mass $m_{d}^{a}$ is projected in $B_{1}$ and the virtual equivalent mass $m_{d}^{c}$ is projected twice about $P_{2}$ and twice about $P_{3}$. About $P_{2} m_{d}^{c}$ is located at a distance $d_{1}=\left\|B_{1} P_{2}\right\|$ from $P_{2}$ perpendicular to line $B_{1} P_{2}$ as illustrated and $m_{d}^{c}$ is located at a distance $a_{23}=\left\|A_{2} P_{2}\right\|$ from $P_{2}$ perpendicular to line $A_{2} P_{2}$ as illustrated. About $P_{3} m_{d}^{c}$ is located at a distance $a_{3}=\left\|A_{2} P_{3}\right\|$ from $P_{3}$ perpendicular to line $A_{2} P_{3}$ as illustrated and $m_{d}^{c}$ is located at a distance $a_{3}^{\prime}=\left\|A_{3} P_{3}\right\|$ from $P_{3}$ perpendicular to line $A_{3} P_{3}$ as illustrated.

The fundamentals of the mass projections are explained in detail in [7, 6]. The virtual equivalent mass $m_{d}^{c}$ determines the positions of the link CoMs of the dyad perpendicular to the lines through the links' joints. To include this property in the triads they have to be projected about each principal point along a closed loop. The closed loop chosen here runs along $B_{1} P_{2} A_{2} P_{3} A_{3}-A_{7} P_{6} A_{6} P_{5} B_{2}$, i.e. via triad 1 , the base, and triad 2 with principal points $P_{5}$ and $P_{6}$ as shown in Fig. 4.

With the method of rotations about the principal joints (RAPJ) [8, 6] DoF 1 and DoF 3 can be analyzed. DoF 1 is the rotational motion of principal element $A_{0} A_{1}$ about principal joint $A_{1}$ with the other two principal elements immobile. This means that only mass $m_{1}$ is moving and for force balance its linear momentum should equal the linear momentum of the total mass of the triad moving along in joint $S_{t r 1}$. The linear momentum of this motion can be written with respect to the aligned reference frame $x_{1} y_{1}$ as

$$
\frac{\bar{L}_{1}}{\dot{\theta}_{1}}=\left[\begin{array}{c}
m_{t r 1} a_{1} \\
0
\end{array}\right]=\left[\begin{array}{c}
m_{1}\left(a_{1}+p_{1}\right) \\
0
\end{array}\right]
$$

with $m_{t r 1}=m_{1}+m_{2}+m_{3}+m_{d}^{a}$ the total mass of the triad model. The resulting force balance condition of this DoF is directly found as

$$
m_{1} p_{1}=\left(m_{2}+m_{3}+m_{d}^{a}\right) a_{1}
$$

DoF 3 is the rotational motion of principal element $A_{2} A_{3}$ about principal joint $A_{2}$ with the other two principal elements immobile and is analyzed similarly as DoF 1 . It is useful to first combine all masses in principal element $A_{2} A_{3}$ as shown in Fig. 2b where the location of the total mass $m_{3}^{\prime}=m_{3}+m_{d}^{c}$ is defined by $e_{3}^{\prime}$ and $f_{3}^{\prime}$ which are calculated as

$$
e_{3}^{\prime}=\frac{m_{3} e_{3}}{m_{3}^{\prime}}, \quad f_{3}^{\prime}=\frac{m_{3} f_{3}+m_{d}^{c} l_{3}}{m_{3}^{\prime}}
$$

Then for the motion of DoF 3 only mass $m_{3}^{\prime}$ is moving and for force balance its linear momentum equals the linear momentum of the total mass of the triad moving along in joint $S_{t r 1}$. The linear momentum of this motion can be written with respect to the aligned reference frame $x_{3} y_{3}$ as 


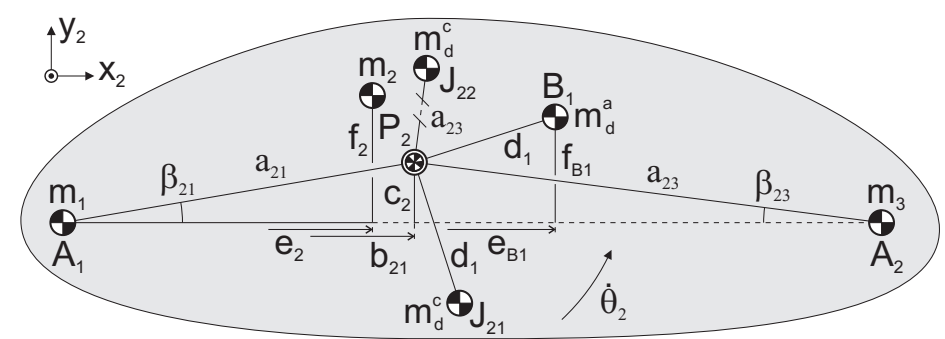

Fig. 3 Equivalent Linear Momentum System of DoF 2 of triad 1 of which $P_{2}$ is the center of mass for force balance.

$$
\frac{\bar{L}_{3}}{\dot{\theta}_{3}}=\left[\begin{array}{c}
m_{t r 1} a_{3} \\
0
\end{array}\right]=\left[\begin{array}{c}
m_{3}^{\prime}\left(a_{3}+p_{3}^{\prime}\right) \\
0
\end{array}\right]
$$

with $p_{3}^{\prime}$ the distance between $m_{3}^{\prime}$ and $P_{3}$ as illustrated. The resulting force balance condition for this DoF is found as

$$
\left(m_{3}+m_{d}^{c}\right) p_{3}^{\prime}=\left(m_{1}+m_{2}+m_{d}^{a}-m_{d}^{c}\right) a_{3}
$$

For DoF 2 the method of rotations about the principal points (RAPP) needs to be used [9, 6]. DoF 2 is the rotational motion of element $A_{1} A_{2}$ about principal point $P_{2}$ with elements $A_{0} A_{1}$ and $A_{2} A_{3}$ solely in translational motion. The linear momentum of this motion must equal zero for force balance since the total mass in joint $S_{t r 1}$ is stationary. To assist formulating the linear momentum equations, the mass motion can be modeled with the Equivalent Linear Momentum System shown in Fig. 3, which is a mass model with the same linear momentum for rotational motion about $P_{2}$. From this model the linear momentum of the motion of DoF 2 can be written with respect to the aligned reference frame $x_{2} y_{2}$ as

$$
\begin{aligned}
\frac{\bar{L}_{2}}{\dot{\theta}_{2}}= & {\left[\begin{array}{l}
0 \\
0
\end{array}\right]=m_{1}\left[\begin{array}{c}
c_{2} \\
-b_{21}
\end{array}\right]+m_{2}\left[\begin{array}{c}
c_{2}-f_{2} \\
-\left(b_{21}-e_{2}\right)
\end{array}\right]+m_{d}^{a}\left[\begin{array}{c}
c_{2}-f_{B 1} \\
-\left(b_{21}-e_{B 1}\right)
\end{array}\right]-} \\
& m_{d}^{c}\left[\begin{array}{c}
b_{21}-e_{B 1} \\
c_{2}-f_{B 1}
\end{array}\right]+m_{3}\left[\begin{array}{c}
c_{2} \\
-\left(b_{21}-l_{2}\right)
\end{array}\right]+m_{d}^{c}\left[\begin{array}{c}
b_{21}-l_{2} \\
c_{2}
\end{array}\right]
\end{aligned}
$$

The resulting force balance conditions for this DoF are directly obtained as

$$
\begin{array}{r}
m_{1} c_{2}+m_{2}\left(c_{2}-f_{2}\right)+m_{d}^{a}\left(c_{2}-f_{B 1}\right)-m_{d}^{c}\left(l_{2}-e_{B 1}\right)+m_{3} c_{2}=0 \\
m_{1} b_{21}+m_{2}\left(b_{21}-e_{2}\right)+m_{d}^{a}\left(b_{21}-e_{B 1}\right)-m_{d}^{c} f_{B 1}+m_{3}\left(b_{21}-l_{2}\right)=0
\end{array}
$$

To calculate the design parameters of the links, when parameters $e_{2}$ and $f_{2}$ are chosen the parameters $b_{21}$ and $c_{2}$ of $P_{2}$ are obtained from Eqs. (8) as 


$$
\begin{aligned}
b_{21} & =\frac{m_{2} e_{2}+m_{d}^{a} e_{B 1}+m_{d}^{c} f_{B 1}+m_{3} l_{2}}{m_{1}+m_{2}+m_{3}+m_{d}^{a}} \\
c_{2} & =\frac{m_{2} f_{2}+m_{d}^{a} f_{B 1}+m_{d}^{c}\left(l_{2}-e_{B 1}\right)}{m_{1}+m_{2}+m_{3}+m_{d}^{a}}
\end{aligned}
$$

Subsequently the design parameters of links $A_{0} A_{1}$ and $A_{2} A_{3}$ can be calculated from the relations

$$
\frac{p_{1}+a_{1}}{a_{1}}=\frac{e_{1}}{b_{1}}=\frac{f_{1}}{c_{1}}, \quad \frac{p_{3}^{\prime}+a_{3}}{a_{3}}=\frac{e_{3}^{\prime}}{b_{3}}=\frac{f_{3}^{\prime}}{c_{3}}
$$

with which the link CoM parameters can be derived to depend on $b_{i}$ and $c_{i}$ as

$$
\begin{aligned}
& e_{1}=\left(\frac{p_{1}}{a_{1}}+1\right) b_{1}=\left(\frac{m_{2}+m_{3}+m_{d}^{a}}{m_{1}}+1\right) b_{1} \\
& f_{1}=\left(\frac{p_{1}}{a_{1}}+1\right) c_{1}=\left(\frac{m_{2}+m_{3}+m_{d}^{a}}{m_{1}}+1\right) c_{1} \\
& e_{3}^{\prime}=\left(\frac{p_{3}^{\prime}}{a_{3}}+1\right) b_{3}=\left(\frac{m_{1}+m_{2}+m_{d}^{a}-m_{d}^{c}}{m_{3}+m_{d}^{c}}+1\right) b_{3} \\
& f_{3}^{\prime}=\left(\frac{p_{3}^{\prime}}{a_{3}}+1\right) c_{3}=\left(\frac{m_{1}+m_{2}+m_{d}^{a}-m_{d}^{c}}{m_{3}+m_{d}^{c}}+1\right) c_{3}
\end{aligned}
$$

The relations for a triad to be mass equivalent with the element $A_{0} A_{3} S_{t r 1}$ are [9]

$$
\begin{array}{cl}
m_{t r_{i}}^{a}\left(l_{1}-b_{1}\right)=m_{t r_{i}}^{b} b_{1}, & m_{t r_{i}}^{c} l_{1}=\left(m_{t r_{i}}^{a}+m_{t r_{i}}^{b}\right) c_{1} \\
m_{t r_{i}}^{a} b_{21}=m_{t r_{i}}^{b}\left(l_{2}-b_{21}\right), & m_{t r_{i}}^{c} l_{2}=\left(m_{t r_{i}}^{a}+m_{t r_{i}}^{b}\right) c_{2} \\
m_{t r_{i}}^{a} b_{3}=m_{t r_{i}}^{b}\left(l_{3}-b_{3}\right), & m_{t r_{i}}^{c} l_{3}=\left(m_{t r_{i}}^{a}+m_{t r_{i}}^{b}\right) c_{3}
\end{array}
$$

with the equivalent triad masses $m_{t r_{1}}^{a}, m_{t r_{1}}^{b}$, and $m_{t r_{1}}^{c}$. With $b_{21}$ and $c_{2}$ known, they are obtained from these relations as

$$
\begin{aligned}
& m_{t r_{1}}^{a}=m_{1}+m_{2}+m_{3}+m_{d}^{a}-m_{t r_{1}}^{b} \\
& m_{t r_{1}}^{b}=\frac{m_{1}+m_{2}+m_{3}+m_{d}^{a}}{l_{2}} b_{21}, \quad m_{t r_{1}}^{c}=\frac{m_{1}+m_{2}+m_{3}+m_{d}^{a}}{l_{2}} c_{2}
\end{aligned}
$$

and subsequently $c_{1}, c_{3}, b_{1}$, and $b_{3}$ can be calculated as

$$
\begin{aligned}
& b_{1}=\frac{m_{t r_{1}}^{a}}{m_{1}+m_{2}+m_{3}+m_{d}^{a}} l_{1}, \quad c_{1}=\frac{m_{t r_{1}}^{c}}{m_{1}+m_{2}+m_{3}+m_{d}^{a}} l_{1} \\
& b_{3}=\frac{m_{t r_{1}}^{b}}{m_{1}+m_{2}+m_{3}+m_{d}^{a}} l_{3}, \quad c_{3}=\frac{m_{t r_{1}}^{c}}{m_{1}+m_{2}+m_{3}+m_{d}^{a}} l_{3}
\end{aligned}
$$

Herewith all parameters are known for force balance. In addition, the similarity parameters of triad 1 defining the shape of element $A_{0} A_{3} S_{t r 1}$ can be found as 


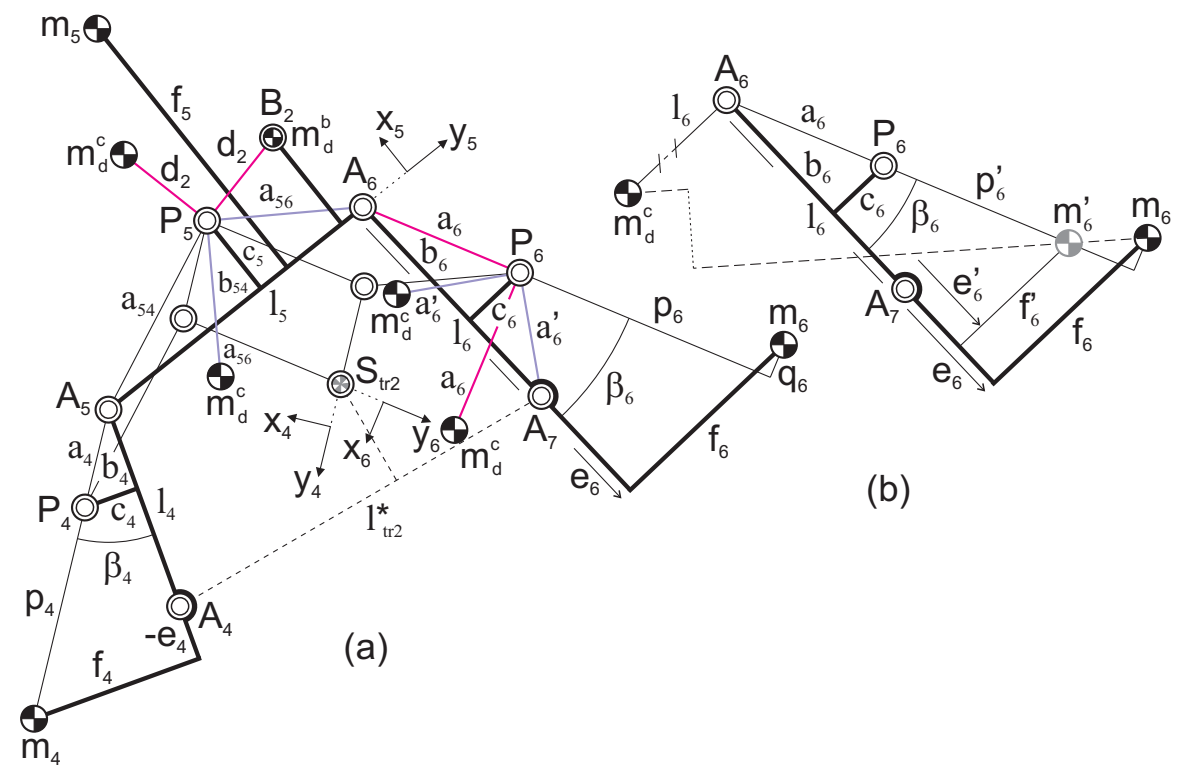

Fig. 4 a) Triad 2 as a 3-DoF principal vector linkage with mass projection of the equivalent dyad with $m_{d}^{b}$ in $B_{2}$ and $m_{d}^{c}$ about $P_{5}$ and $P_{6}$; b) For analysis of DoF 3 the masses in link $A_{6} A_{7}$ can be combined as $m_{6}^{\prime}$.

$$
\lambda_{t r 11}=\frac{m_{t r_{1}}^{b}}{m_{1}+m_{2}+m_{3}+m_{d}^{a}}, \quad \lambda_{t r 12}=\frac{m_{t r_{1}}^{c}}{m_{1}+m_{2}+m_{3}+m_{d}^{a}}
$$

and the principal dimensions $a_{1}, a_{21}, a_{23}$, and $a_{3}$ can be calculated as

$$
\begin{aligned}
a_{1} & =\sqrt{b_{1}^{2}+c_{1}^{2}}=l_{1} \sqrt{\left(1-\lambda_{t r 11}\right)^{2}+\lambda_{t r 12}^{2}} \\
a_{21} & =\sqrt{b_{21}^{2}+c_{2}^{2}}=l_{2} \sqrt{\lambda_{t r 11}^{2}+\lambda_{t r 12}^{2}} \\
a_{23} & =\sqrt{\left(l_{2}-b_{21}\right)^{2}+c_{2}^{2}}=l_{2} \sqrt{\left(1-\lambda_{t r 11}\right)^{2}+\lambda_{t r 12}^{2}} \\
a_{3} & =\sqrt{b_{3}^{2}+c_{3}^{2}}=l_{3} \sqrt{\lambda_{t r 11}^{2}+\lambda_{t r 12}^{2}}
\end{aligned}
$$

For triad 2 the force balance conditions and the design parameters are derived similarly to triad 1 . Figure 4 a shows the triad $A_{4} A_{5} A_{6} A_{7}$ as a $3-\mathrm{DoF}$ principal vector linkage with a principal point $P_{4}, P_{5}$, and $P_{6}$ in each of the three principal elements. These principal points define together with the principal joints $A_{5}$ and $A_{6}$ the three illustrated parallelograms which trace the common CoM in $S_{t r 2}$ for all motion. The lengths of the sides of the parallelograms are the principal dimensions $a_{4}, a_{54}, a_{56}$, and $a_{6}$.

In Fig. 4a also the equivalent masses $m_{d}^{b}$ and $m_{d}^{c}$ of the dyad are projected with the real equivalent mass $m_{d}^{b}$ in $B_{2}$ and the virtual equivalent mass $m_{d}^{c}$ projected twice 


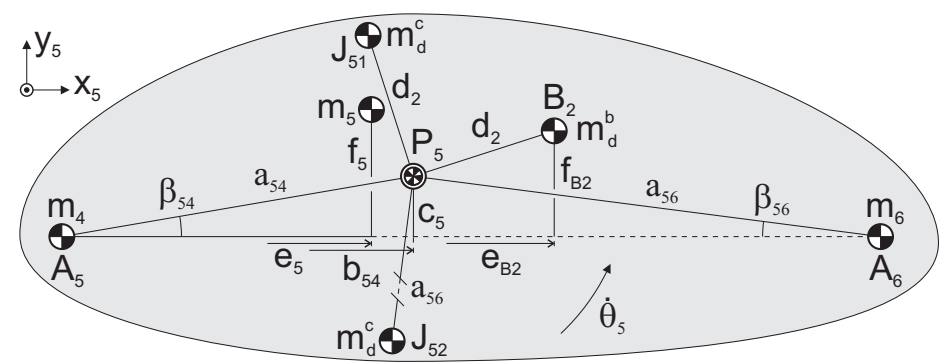

Fig. 5 Equivalent Linear Momentum System of DoF 2 of triad 2 of which $P_{5}$ is the center of mass for force balance.

about $P_{5}$ and twice about $P_{6}$. About $P_{5} m_{d}^{c}$ is located at a distance $d_{2}=\left\|B_{2} P_{5}\right\|$ from $P_{5}$ perpendicular to line $B_{2} P_{5}$ as illustrated and $m_{d}^{c}$ is located at a distance $a_{56}=\left\|A_{6} P_{5}\right\|$ from $P_{5}$ perpendicular to line $A_{6} P_{5}$ as illustrated. About $P_{6} m_{d}^{c}$ is located at a distance $a_{6}=\left\|A_{6} P_{6}\right\|$ from $P_{6}$ perpendicular to line $A_{6} P_{6}$ as illustrated and $m_{d}^{c}$ is located at a distance $a_{6}^{\prime}=\left\|A_{7} P_{6}\right\|$ from $P_{6}$ perpendicular to line $A_{7} P_{6}$ as illustrated.

Following the same procedure as for triad 1, the linear momentum of DoF 1 of triad 2, which is the rotational motion of $A_{4} A_{5}$ about $A_{5}$ with the other triad links immobile, can be written relative to the aligned reference frame $x_{4} y_{4}$ as

$$
\frac{\bar{L}_{4}}{\dot{\theta}_{4}}=\left[\begin{array}{c}
m_{t r 2} a_{4} \\
0
\end{array}\right]=\left[\begin{array}{c}
m_{4}\left(a_{4}+p_{4}\right) \\
0
\end{array}\right]
$$

with $m_{t r 2}=m_{4}+m_{5}+m_{6}+m_{d}^{b}$ the total mass of the triad model with CoM in joint $S_{t r 2}$. The masses in principal element $A_{6} A_{7}$ can be combined in total mass $m_{6}^{\prime}=m_{6}+m_{d}^{c}$ with CoM defined by $e_{6}^{\prime}$ and $f_{6}^{\prime}$ which are calculated as

$$
e_{6}^{\prime}=\frac{m_{6} e_{6}}{m_{6}^{\prime}}, \quad f_{6}^{\prime}=\frac{m_{6} f_{6}-m_{d}^{c} l_{6}}{m_{6}^{\prime}}
$$

Then the linear momentum of DoF 3 of triad 2, which is the rotational motion of principal element $A_{6} A_{7}$ about principal joint $A_{6}$ with the other two principal elements immobile, can be written relative to the aligned reference frame $x_{6} y_{6}$ as

$$
\frac{\bar{L}_{6}}{\dot{\theta}_{6}}=\left[\begin{array}{c}
m_{t r 2} a_{6} \\
0
\end{array}\right]=\left[\begin{array}{c}
m_{6}^{\prime}\left(a_{6}+p_{6}^{\prime}\right) \\
0
\end{array}\right]
$$

For DoF 2, the rotational motion of element $A_{5} A_{6}$ about principal point $P_{5}$ with elements $A_{4} A_{5}$ and $A_{6} A_{7}$ solely in translational motion, Fig. 5 shows the Equivalent Linear Momentum System. For force balance $P_{5}$ is the CoM of this mass model. From this model the linear momentum can be written with respect to the aligned reference frame $x_{5} y_{5}$ as 
Force Balance Conditions with Mass Equivalent Modeling

$$
\begin{aligned}
\frac{\bar{L}_{5}}{\dot{\theta}_{5}}= & {\left[\begin{array}{l}
0 \\
0
\end{array}\right]=m_{4}\left[\begin{array}{c}
c_{5} \\
-b_{54}
\end{array}\right]+m_{5}\left[\begin{array}{c}
c_{5}-f_{5} \\
-\left(b_{54}-e_{5}\right)
\end{array}\right]+m_{d}^{b}\left[\begin{array}{c}
c_{5}-f_{B 2} \\
-\left(b_{54}-e_{B 2}\right)
\end{array}\right]+} \\
& m_{d}^{c}\left[\begin{array}{c}
b_{54}-e_{B 2} \\
c_{5}-f_{B 2}
\end{array}\right]+m_{6}\left[\begin{array}{c}
c_{5} \\
-\left(b_{54}-l_{5}\right)
\end{array}\right]-m_{d}^{c}\left[\begin{array}{c}
b_{54}-l_{5} \\
c_{5}
\end{array}\right]
\end{aligned}
$$

From Eqs. (17), (19) and (20) the force balance conditions for triad 2 are obtained as

$$
\begin{aligned}
m_{4} p_{4} & =\left(m_{5}+m_{6}+m_{d}^{b}\right) a_{4} \\
\left(m_{6}+m_{d}^{c}\right) p_{6}^{\prime} & =\left(m_{4}+m_{5}+m_{d}^{b}-m_{d}^{c}\right) a_{6} \\
0 & =m_{4} c_{5}+m_{5}\left(c_{5}-f_{5}\right)+m_{d}^{b}\left(c_{5}-f_{B 2}\right)+m_{d}^{c}\left(l_{5}-e_{B 2}\right)+m_{6} c_{5} \\
0 & =m_{4} b_{54}+m_{5}\left(b_{54}-e_{5}\right)+m_{d}^{b}\left(b_{54}-e_{B 2}\right)+m_{d}^{c} f_{B 2}+m_{6}\left(b_{54}-l_{5}\right)
\end{aligned}
$$

Similarly as for triad 1 , the parameters of the principal points $P_{4}, P_{5}$, and $P_{6}$ can be calculated as

$$
\begin{aligned}
b_{4} & =\frac{m_{t r_{2}}^{a}}{m_{4}+m_{5}+m_{6}+m_{d}^{b}} l_{4}, & c_{4} & =\frac{m_{t r_{2}}^{c}}{m_{4}+m_{5}+m_{6}+m_{d}^{b}} l_{4} \\
b_{54} & =\frac{m_{5} e_{5}+m_{d}^{b} e_{B 2}-m_{d}^{c} f_{B 2}+m_{6} l_{5}}{m_{4}+m_{5}+m_{6}+m_{d}^{b}}, & c_{5} & =\frac{m_{5} f_{5}+m_{d}^{b} f_{B 2}-m_{d}^{c}\left(l_{5}-e_{B 2}\right)}{m_{4}+m_{5}+m_{6}+m_{d}^{b}} \\
b_{6} & =\frac{m_{t r_{2}}^{b}}{m_{4}+m_{5}+m_{6}+m_{d}^{b}} l_{6}, & c_{6} & =\frac{m_{t r_{2}}^{c}}{m_{4}+m_{5}+m_{6}+m_{d}^{b}} l_{6}
\end{aligned}
$$

with

$$
\begin{aligned}
& m_{t r_{2}}^{a}=m_{4}+m_{5}+m_{6}+m_{d}^{b}-m_{t r_{2}}^{b} \\
& m_{t r_{2}}^{b}=\frac{m_{4}+m_{5}+m_{6}+m_{d}^{b}}{l_{5}} b_{54}, \quad m_{t r_{2}}^{c}=\frac{m_{4}+m_{5}+m_{6}+m_{d}^{b}}{l_{5}} c_{5}
\end{aligned}
$$

and subsequently the parameters of the CoMs of the links can be calculated with

$$
\begin{array}{ll}
e_{4}=\frac{m_{4}+m_{5}+m_{6}+m_{d}^{b}}{m_{4}} b_{4}, & f_{4}=\frac{m_{4}+m_{5}+m_{6}+m_{d}^{b}}{m_{4}} c_{4} \\
e_{6}^{\prime}=\frac{m_{4}+m_{5}+m_{6}+m_{d}^{b}}{m_{6}+m_{d}^{c}} b_{6}, & f_{6}^{\prime}=\frac{m_{4}+m_{5}+m_{6}+m_{d}^{b}}{m_{6}+m_{d}^{c}} c_{6} \\
e_{6}=\frac{m_{6}+m_{d}^{c}}{m_{6}} e_{6}^{\prime}, & f_{6}=\frac{\left(m_{6}+m_{d}^{c}\right) f_{6}^{\prime}+m_{d}^{c} l_{6}}{m_{6}}
\end{array}
$$

Herewith all parameters are known for force balance of triad 2. The similarity parameters of triad 2 which define the shape of element $A_{4} A_{7} S_{t r 2}$ can be found as

$$
\lambda_{t r 21}=\frac{m_{t r_{2}}^{b}}{m_{4}+m_{5}+m_{6}+m_{d}^{b}}, \quad \lambda_{t r 22}=\frac{m_{t r_{2}}^{c}}{m_{4}+m_{5}+m_{6}+m_{d}^{b}}
$$


and the principal dimensions $a_{4}, a_{54}, a_{56}$, and $a_{6}$ can be calculated as

$$
\begin{aligned}
a_{4} & =\sqrt{b_{4}^{2}+c_{4}^{2}}=l_{4} \sqrt{\left(1-\lambda_{t r 21}\right)^{2}+\lambda_{t r 22}^{2}} \\
a_{54} & =\sqrt{b_{54}^{2}+c_{5}^{2}}=l_{5} \sqrt{\lambda_{t r 21}^{2}+\lambda_{t r 22}^{2}} \\
a_{56} & =\sqrt{\left(l_{5}-b_{54}\right)^{2}+c_{5}^{2}}=l_{5} \sqrt{\left(1-\lambda_{t r 21}\right)^{2}+\lambda_{t r 22}^{2}} \\
a_{6} & =\sqrt{b_{6}^{2}+c_{6}^{2}}=l_{6} \sqrt{\lambda_{t r 21}^{2}+\lambda_{t r 22}^{2}}
\end{aligned}
$$

\section{Discussion and conclusion}

In this paper it was shown how with mass equivalent modeling the force balance conditions can be derived of a complex multi-degree-of-freedom parallel mechanism with multiple closed loops of which one depends on the other closed loops. By dividing the mechanism in three parts it was investigated as a combination of a mass equivalent dyad on top of two mass equivalent triads. With the method of principal vectors and the linear momentum equations of each relative degree-of-freedom the force balance conditions were derived and the design parameters were calculated for each of three parts individually.

The approach of mass equivalent modeling can also be used for the synthesis of complex force balanced mechanisms by composing the new mechanism of combined mass equivalent linkages. There is a wide variety of possibilities to do this, already when solely using mass equivalent dyads and triads. The advantage with mass equivalent modeling is that the closed-loop relations do not need to be formulated but are considered implicitly. Also it is possible to apply this method for spatial mechanisms. For instance by applying two mass equivalent triads as balanced fourbar linkages with common base as in this paper but placing them in different planes under a relative angle instead of having them in the same plane, with a spatially moving mass equivalent dyad on top.

Acknowledgements This publication was financially supported by the Niels Stensen Fellowship.

\section{References}

1. Berkof, R.S., Lowen, G.G.: A new method for completely force balancing simple linkages. Engineering for Industry pp. 21-26 (1969)

2. Brown, G.W.: Suspension system for supporting and conveying equipment, such as a camera, patent US-4710819 (1987)

3. Chung, W.K., Cho, H.S.: On the dynamic characteristics of a balanced PUMA-760 robot. Industrial Electronics 35(2), 222-230 (1988) 
4. Gosselin, C.M., Vollmer, F., Côté, G., Wu, Y.: Synthesis and design of reactionless threedegree-of-freedom parallel mechanisms. IEEE Transactions on Robotics and Automation 20(2), 191-199 (2004)

5. Ishida, K., Matsuda, T.: Performance characteristics and working comfortableness of forest workers of a new non-vibrating chain saw utilizing perfectly balanced rotation-reciprocation device. Proceedings of the Fifth World Congress of Theory of Machines and Mechanisms, ASME pp. 951-954 (1979)

6. Van der Wijk, V.: Methodology for analysis and synthesis of inherently force and momentbalanced mechanisms - theory and applications (dissertation). University of Twente (free download: http://dx.doi.org/10.3990/1.9789036536301) (2014)

7. Van der Wijk, V.: Closed-chain principal vector linkages. In: P. Flores and F. Viadero (eds.), New Trends in Mechanism and Machine Science 24, 829-837 (2015). Springer.

8. Van der Wijk, V.: Mass equivalent dyads. In: S. Bai and M. Ceccarrelli (eds.), Recent Advances in Mechanism Design for Robotics MMS 33, 35-45 (2015). Springer.

9. Van der Wijk, V.: Mass equivalent triads. Proceedings of the 14th IFToMM World Congress in Mechanism and Machine Science p. OS13.131/DOI 10.6567 (2015)

10. Van der Wijk, V., Krut, S., Pierrot, F., Herder, J.L.: Design and experimental evaluation of a dynamically balanced redundant planar 4-RRR parallel manipulator. I.J. of Robotics Research 32(6), 744-759 (2013)

11. Wu, Y., Gosselin, C.M.: Design of reactionless 3-DOF and 6-DOF parallel manipulators using parallelepiped mechanisms. IEEE Transactions on Robotics 21(5), 821-833 (2005)

12. Wu, Y., Gosselin, C.M.: On the dynamic balancing of multi-dof parallel mechanisms with multiple legs. Mechanical Design 129, 234-238 (2007) 